\title{
Multi-point observations of intermittency in the cusp regions
}

\author{
M. M. Echim ${ }^{1,3}$, H. Lamy ${ }^{1}$, and T. Chang ${ }^{2}$ \\ ${ }^{1}$ Belgian Institute for Space Aeronomy, Avenue Circulaire 3, 1180 Bruxelles, Belgium \\ ${ }^{2}$ Kavli Institute for Astrophysics and Space Research, M. I. T., Cambridge, MA 02139, USA \\ ${ }^{3}$ Institute for Space Sciences, Atomistilor 409, 077125 Bucharest, Romania
}

Received: 25 April 2007 - Revised: 9 August 2007 - Accepted: 12 August 2007 - Published: 17 August 2007

\begin{abstract}
In this paper we investigate the statistical properties of magnetic field fluctuations measured by the four Cluster spacecraft in the cusp and close to the interface with the magnetospheric lobes, magnetopause and magnetosheath. At lower altitudes along the outbound orbit of 26 February 2001, the magnetic field fluctuations recorded by all four spacecraft are random and their Probability Distribution Functions (PDFs) are Gaussian at all scales. The flatness parameter, $\mathcal{F}$ - related to the kurtosis of the time series, is equal to 3. At higher altitudes, in the cusp and its vicinity, closer to the interface with the magnetopause and magnetosheath, the PDFs from all Cluster satellites are non-Gaussian and show a clear intermittent behavior at scales smaller than $\tau_{G} \approx 61 \mathrm{~s} \mathrm{(or}$ $170 \mathrm{~km})$. The flatness parameter increases to values greater than 3 for scales smaller than $\tau_{G}$. A Haar wavelet transform enables the identification of the "events" that produce sudden variations of the magnetic field and of the scales that have most of the power. The LIM parameter (i.e. normalized wavelet power) indicates that events for scales below $65 \mathrm{~s}$ are non-uniformly distributed throughout the cusp passage. PDFs, flatness and wavelet analysis show that at coarsegrained scales larger than $\tau_{G}$ the intermittency is absent in the cusp. Fluctuations of the magnetic energy observed during the same orbit in the magnetosheath show PDFs that tend toward a Gaussian at scales smaller than $\tau_{G}$ found in the cusp. The flatness analysis confirms the decreasing of $\tau_{G}$ from cusp to magnetosheath. Our analysis reveals the turbulent cusp as a transition region from a non-intermittent turbulent state inside the magnetosphere to an intermittent turbulent state in the magnetosheath that has statistical properties resembling the solar wind turbulence. The observed turbulent fluctuations in the cusp suggests a phenomenon of nonlinear interactions of plasma coherent structures as in contemporary models of space plasma turbulence.
\end{abstract}

Correspondence to: M. M. Echim

(marius.echim@oma.be)

\section{Introduction}

The terrestrial plasma and magnetic field are in continuous contact with the interplanetary magnetic field and solar wind plasma. Energy, mass and momentum are transported from the solar wind to the magnetosphere where they are redistributed and the input energy is eventually dissipated by the system of electric currents. Extensive observations at the interface between the magnetosphere and the shocked solar wind reveal fluctuations of the plasma variables and electromagnetic field over a broad range of scales.

Intermittency is related to the sudden occurrence, in space or time, of large amplitude variations of plasma variables such as the bulk velocity or magnetic field intensity. In the framework of the classical paradigm of the Richardson cascade of turbulent transfer of energy, intermittency occurs when the coherent turbulent structures (eddies) are not space filling at all scales or when the energy transfer rate between scales is not constant but varies intermittently. Intermittency of plasma velocity and magnetic field fluctuations has been observed in the solar wind on a broad range of radial distances, see, for instance, Burlaga (1991); Burlaga and F.-Vinãs (2004), Marsch and Tu (1994), Ruzmaikin et al. (1995), Pagel and Balogh (2001), Hnat et al. (2002) Bruno et al. (2001, 2003), Sorriso-Valvo et al. (2001), Leubner (2004), Leubner and Vörös (2005). Intermittency has been also detected in the terrestrial magnetosphere, particularly in the plasma sheet and magnetotail (Lui, 2001; Vörös et al., 2003; Weygand et al., 2005; Consolini et al., 2005).

The investigation of turbulence in the magnetospheric cusps has been enhanced by observations from such satellites like Interball, Polar and most recently Cluster. Evidence of magnetic turbulence has been identified over a broad range of frequencies (from $1 \mathrm{~Hz}$ to beyond electron cyclotron frequency) of the B-field spectra, in the so-called Turbulent Boundary Layer (Savin et al., 1998, 2002; Pickett et al., 1999, 2002). Case studies of magnetic field fluctuations in

Published by Copernicus Publications on behalf of the European Geosciences Union and the American Geophysical Union. 
the cusp show magnetic field fluctuations with various degrees of intermittency (Yordanova et al., 2005). The power spectrum of the magnetic turbulence in the Northern cusp during Northward IMF show breaks in the vicinity of the local ion cyclotron frequency (Yordanova et al., 2005; Nykyri et al., 2006). Coherent, vortex-like Alfvenic structures have been recently detected in the cusp by the Cluster quartet (Sundkvist et al., 2005) and might be considered as responsible for the intermittency at small scales. Nevertheless, a global picture of the origin of cusp intermittency has not yet emerged.

In this paper we investigate some of the statistical properties of the magnetic energy fluctuations observed by Cluster on the outbound leg of the orbit of 26 February 2001. We analyze high resolution magnetic field measurements from the FGM experiment (Balogh et al., 2001). The interval of analysis encompasses lower altitude magnetospheric regions, the Northern magnetospheric cusp and the magnetosheath. It is thus possible to study fluctuations from large, driving scales, to small, kinetic dissipation scales and to obtain information about the physics of the cascade of energy transfer in the cusp, and at altitudes below and above.

In the next section we briefly describe the methods of analysis. In the third section we show the main results of an analysis of B-field fluctuations in the magnetosphere, cusp and magnetosheath. The paper is summarized in the last section where we also discuss the relevance of our results in the context of space plasma turbulence models.

\section{Methods of analysis: PDFs, scaling, flatness, wavelet transform}

The spatial structure of turbulence may be investigated by computing differences between any two points separated by the distance $\delta x$. In space plasmas, variables such as speed or magnetic field intensity are sampled by satellite measurements along the orbit. Thus, space differences are obtained from time differences of the time series $Q$ :

$\Delta Q_{\tau}=Q(t+\tau)-Q(t)$

where $Q$ stands for any plasma variable (e.g. bulk velocity, $Q \equiv V$, or magnetic field intensity, $Q \equiv B$ ). Time differences are equivalent to space differences when the Taylor hypothesis (Taylor, 1938) is valid, i.e. a turbulent structure, like an eddy, transits the spacecraft at a time which is small compared to its own time evolution. In this case the frequency spectra are related to wave number spectra (Matthaeus and Goldstein, 1982).

The normalized histogram of the ensemble of time differences obtained with (1) over an interval $\left[t_{1}, t_{2}\right]$ gives $P(\tau)$, the Probability Distribution Function (PDF) of the fluctuations of $Q$ at scale $\tau$. Intermittency is usually evidenced as a departure of the PDFs from a Gaussian distribution and is recognized as the process responsible for increased probabilities of large amplitude fluctuations at smaller scales.

The PDF of a Gaussian random variable is self-similar (or self-affine), i.e. the PDFs scale as a power law and collapse on a single master curve after a suitable change of variable (Sornette, 2000). Non-Gaussian PDFs can also have selfsimilar scaling properties. A mono-power law for Levytype distributions has been proposed by Hnat et al. (2002) to rescale PDFs obtained for the fluctuations of $B^{2}$ measured in the solar wind:

$P\left(\Delta Q_{\tau}, \tau\right)=\tau^{-\alpha} P_{s}\left(\tau^{-\alpha} \Delta Q_{\tau}, \tau\right)$

where $Q=B^{2}$ and $\alpha$ is the mono-power scaling exponent (Hnat et al., 2002) computed from $P_{s}$, the non-scaled PDFs. The scaling law (2) has been tested for magnetic field fluctuations in the cusp and provided partial collapse of B-field PDFs (Yordanova et al., 2005).

A more quantitative estimation of the intermittent character of a time series can be obtained by computing the flatness parameter at a scale $\tau$ (Meneveau, 1991; Frisch, 1995; Bruno et al., 2003)

$\mathcal{F}=\frac{<\Delta Q_{\tau}^{4}>}{<\Delta Q_{\tau}^{2}>^{2}}$

where $\left\langle\Delta Q_{\tau}^{p}\right\rangle, p=2,4$ is the structure function of order $\mathrm{p}$ of the time series $Q(t)$ and $<>$ denotes averaging over all points. A given time series is intermittent if $\mathcal{F}$ continually increases at smaller scales. Another time series will be said more intermittent if $\mathcal{F}$ grows faster. The scale at which $\mathcal{F}$ starts to grow defines the lower limit of the inertial range. Gaussian fluctuations constitute a special case of self-similar, non-intermittent signals. They produce a value of $\mathcal{F}$ equal to 3.

Turbulence is generally seen as the co-existence and interaction of coherent structures at multiple scales (Frisch, 1995; Chang, 1998). It is therefore important to identify the scales of structures that concentrate most of the power and where the interactions are localized. Although a non-Gaussian PDF may indicate the presence of intermittency it does not tell anything about the localization, in time/space, of the "events" that produce large amplitude fluctuations and at which scales is concentrated the power of these intermittent fluctuations. Windowed Fourier analysis or wavelet transform can give insight about the distribution in time/space of the power corresponding to each scale. As pointed out by Bruno et al. (2001), the wavelet transform is more appropriate to study turbulence since the wavelet modes give information about the spatial structures, eddies or coherent structures, of the plasma flow. Given a "mother" wavelet function, $\Psi(x)$, the wavelet coefficients of the signal $Q(t)$ are obtained as the convolution between $Q(t)$ and all the possible scaled and translated instances of $\Psi$ (Torrence and Compo (1998), for an introductory review see Daubechies (1992)):

$C(\tau, t)=\frac{1}{\tau} \int_{t_{1}}^{t_{2}} \Psi\left(\frac{t^{\prime}-t}{\tau}\right) Q\left(t^{\prime}\right) d t^{\prime}$. 
Note that $|C(\tau, t)|^{2}$ is a measure of the energy of the field $Q(t)$ at the given scale and position (when the Taylor hypothesis is satisfied). The local intermittency measure (LIM) or normalized power is derived from wavelet coefficients (Farge et al., 1990) as below:

$$
\operatorname{LIM}(\tau, t)=\frac{|C(\tau, t)|^{2}}{<|C(\tau, t)|^{2}>}
$$

where the average $<>$ is computed over the entire time interval $\left[t_{1}, t_{2}\right]$. Wavelet transform was used to investigate turbulence in the cusp (Yordanova et al., 2004; Sundkvist et al., 2005) and solar wind (Bruno et al., 2001), or to investigate the role of intermittent electric fields in the auroral zone (Tam et al., 2005).

\section{Intermittency in the cusp; Cluster data analysis}

In this study we use high resolution magnetic field data from the FGM experiment on-board the four Cluster satellites (Balogh et al., 2001). Data were made available through ESA's Cluster Active Archive on-line database (http://caa. estec.esa.int). The FGM instrument provided B-field measurements with $66 \mathrm{~Hz}$ burst mode resolution, thus the time series investigated in this study comprise more than $10^{6}$ samples.

\subsection{Overview of cluster and geophysical data}

On 26 February 2001 Cluster satellites spent about two hours in the cusp and its adjacent regions, between approximately 04:00 UT and 06:10 UT. The plasma state during this cusp passage is well documented in previous studies (Lundin et al., 2003; Taylor et al., 2004). We summarize here the facts relevant for our analysis of intermittent fluctuations. The Cluster quartet moved from the northern lobe into the cusp at about 04:02 UT (Taylor et al., 2004). The subsequent two hours of data show a complex intermixing of plasmas with different properties: low temperature, high density magnetosheath-like plasma, and higher temperature, quasi trapped magnetospheric-like plasma. Large scale structures observed in ion and electron energy spectra were interpreted as hallmarks of global motion of the cusp (Taylor et al., 2004) while smaller scale events observed between 05:1005:30 UT in ion energy spectra were assigned to plasma elements impulsively penetrating from the magnetosheath (Lundin et al., 2003).

During the first six hours of 26 February 2001 the auroral indices showed low levels of activity. However the AEindex increases to values greater than $100 \mathrm{nT}$ immediately after 06:30 UT; it stays above this threshold during the rest of the day with a peak of activity between 18:00-24:00 UT. Magnetic field data from ACE satellite mapped to $1 \mathrm{AU}$, indicate an IMF $B_{z}$ turning Southward at about 04:00 UT and remaining negative until 10:30 UT. Data from the Ultraviolet

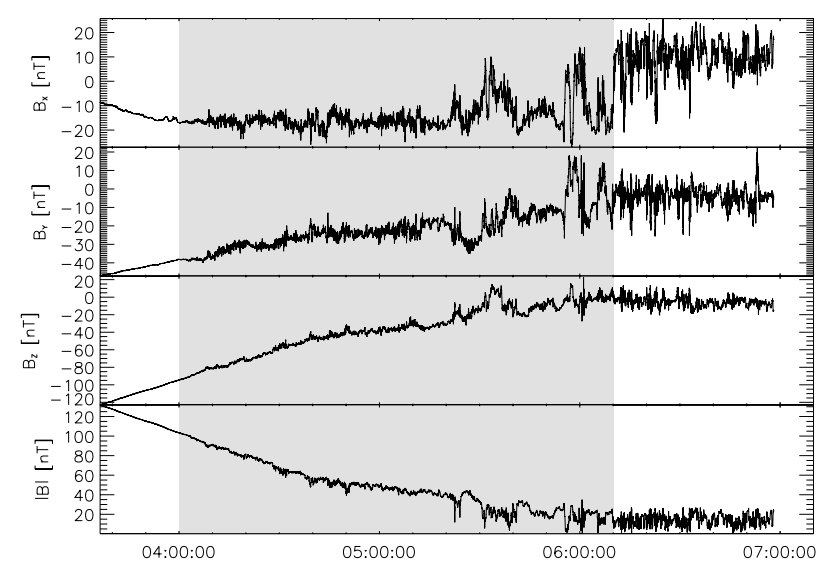

Fig. 1. The components and intensity of the magnetic field measured by Cluster-1 on 26 February, 2001. The interval corresponding to the traversal of cusp regions has been shaded in gray.

Imager on-board POLAR satellite (available through the web interface http://csds.uah.edu) indicate auroral activations in the evening sector throughout the day. It seems that the Cluster cusp passage reported in this study took place less than half an hour before a sustained substorm magnetospheric activity, thus it provides a snapshot through the magnetospheric cusp prior to the onset of a substorm.

The time series of magnetic field data were divided in three subintervals: (A) from 03:36:00 UT to 04:00:00 UT, corresponding to the magnetospheric lobe; (B) from 04:00:00 UT to $06: 10: 00 \mathrm{UT}$, corresponding to the turbulent cusp and adjacent regions; (C) from 06:10:00 UT to 07:00:00 UT, corresponding to the magnetosheath. The bow shock traversal and exit in the solar wind took place at 09:45:00 UT. Magnetic field data from Cluster 1, comprising all three intervals defined above, are shown in Fig. 1. It is found that the fluctuations of $|B|^{2}$ in each of the intervals (A), (B) or (C) have specific statistical properties. This quantitative analysis provides a complementary view to the studies devoted previously to this Cluster cusp passage.

\subsection{Normalization and scaling}

In order to remove/detrend the large scale variations due to satellite traversal of the main, decreasing, geomagnetic field, the dipole component (which is dominant at lower altitudes as shown by Fig. 1) may be extracted by changing the frame of reference to a mean B-field aligned frame (Yordanova et al., 2005) or by subtracting a linear fit of the time series (Nykyri et al., 2004). Another possible approach is to subtract from the measured field the components of the Tsyganenko model (Tsyganenko, 1995); our preliminary test of this approach is not totally satisfactory due to the systematic overestimation by the Tsyganenko model of the B-field intensity in the cusp (Tsyganenko and Russell, 1999). 


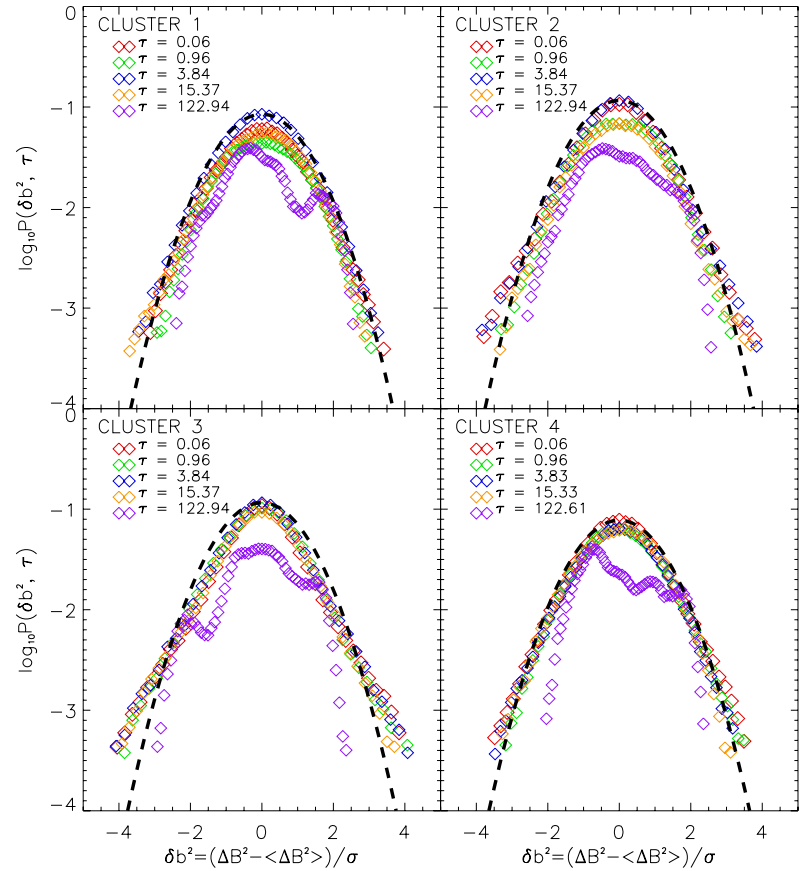

Fig. 2. PDFs of the fluctuations of the magnetic field energy $\left(|B|^{2}\right)$ measured in the magnetosphere by the four Cluster satellites; the PDFs have been rescaled with respect to their $\sigma$. The scales $\tau$ are color coded and given in seconds.

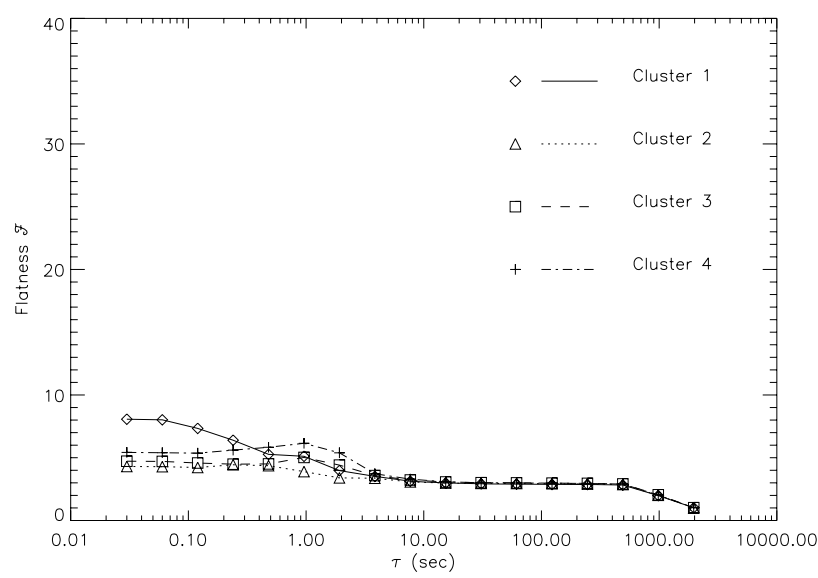

Fig. 3. Flatness $\mathcal{F}$ versus time scale $\tau$ for the magnetic energy fluctuations measured in the magnetosphere by the four Cluster satellites.

An implicit, partial, removal of the dipole component can be also achieved by computing first the differences $\Delta B^{2}(t, \tau)$ from the actual (non de-trended) data; subsequently the mean value of the fluctuations at each scale is subtracted and a new "ensemble" of fluctuations, $\Delta B^{2}(t, \tau)-<\Delta B^{2}(t, \tau)>$, is then constructed, where the symbol " $<>$ " means average over the whole time series. In addition to subtraction of the mean value, each ensemble of time differences is divided by its own variance, $\sigma(\tau)$. Thus the PDFs can be computed for the new set of fluctuations:

$\delta b^{2}=\frac{\Delta B^{2}(t, \tau)-<\Delta B^{2}(t, \tau)>}{\sigma(\tau)}$

where differences $\Delta B^{2}(t, \tau)$ have been computed with (1) by moving an overlapping window of width $\tau=2^{j} \delta t$, over the entire time interval; $\delta t$ is the time resolution of the measurements $(\delta t=0.015 \mathrm{~s})$ and $j=\overline{1,2, \ldots 15}$. This procedure is equivalent to a parametric rescaling technique akin to the finite size rescaling method that has been applied to a wide variety of nonlinear studies (Sornette, 2000).

When the scaling defined in (6) is applied to a Gaussian variable its PDFs at various scales collapse on a single master curve. Other finite size scaling laws have been postulated, for instance for log-normal (Castaing et al., 1990) or Levy PDFs (Hnat et al., 2002). The scaling of PDFs provides important information about the process of energy transfer, like selfsimilarity, space filling properties and intermittency of the energy transfer rate. Note also that a self-similar transfer of energy corresponds to a perfect rescaling of the PDFs by a simple, one-parameter law.

\subsection{Magnetospheric lobe}

A selection of the PDFs obtained for 5 of the 15 investigated scales are shown in figure 2, for all the four Cluster satellites. The PDFs virtually collapse on a same curve and they show Gaussian behavior at all scales. There are no intermittent fluctuations in the magnetospheric lobes during the analyzed interval. We do not see significant differences between the four spacecraft except perhaps for Cluster 3 that show a faint departure from Gaussianity. Note that Cluster 3 is the "frontend" of the thetraedron in the Earth-Sun direction, thus it is likely that it is the first to encounter structures traveling in the anti-sunward direction. The asymmetry obtained for the central part of PDFs at the largest scale (122.94 s) is due to the dipolar component of magnetic field, which is strong and incompletely removed in the lobe by the implicit procedure (6).

The flatness has also been computed for the four spacecraft and the results are shown in Fig. 3. It is nearly constant at all considered scales and has values close to 3 , as expected from nearly Gaussian fluctuations. The small values of $\mathcal{F}$ at virtually all scales in the magnetosphere confirms the absence of intermittency. The interval A has been analyzed mainly as a test of the analysis method and an illustration of Gaussian PDFs obtained for experimental data a-priori known to be non-intermittent.

\subsection{Cusp and adjacent regions}

In the northern cusp regions, between 04:00:00 UT and 06:10:00 UT, the magnetic field fluctuations have statistical 


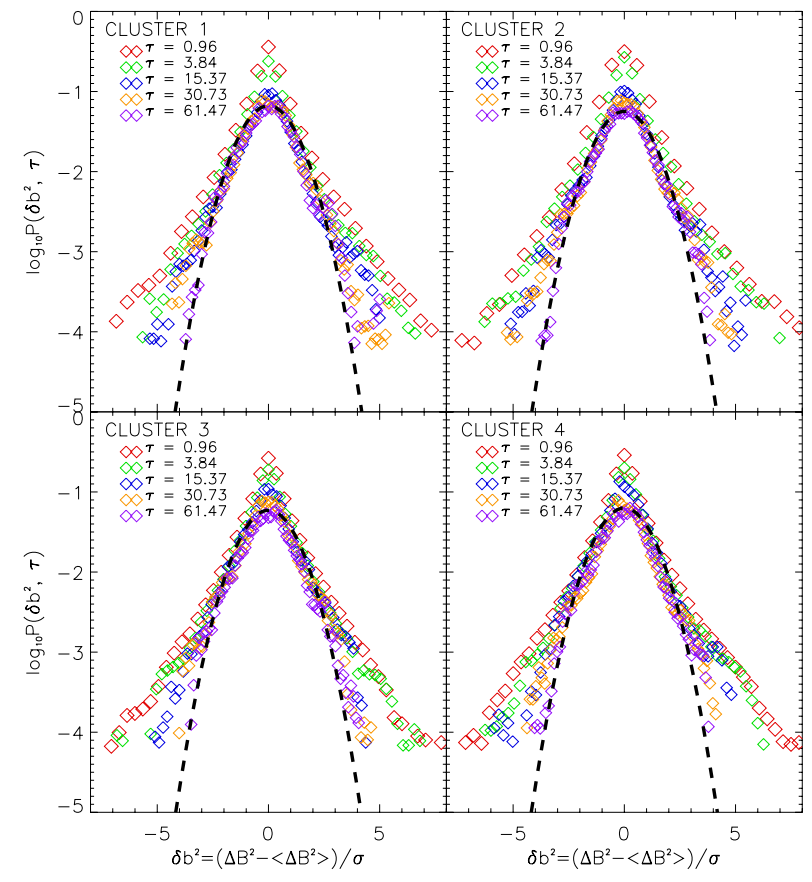

Fig. 4. PDFs of the magnetic energy fluctuations measured by the four Cluster satellites in the cusp; the PDFs have been scaled with respect to their variance. The scales $\tau$ are color coded and given in seconds.

properties significantly different than in the lobe. Figure 4 shows a selection of PDFs obtained for $|B|^{2}$ fluctuations and rescaled with respect to their variance, as described in the previous section. The probability distribution functions were computed for magnetic field data from all the four Cluster satellites, thus giving a multi-point picture of the magnetic energy fluctuations in the cusp. The PDFs depart significantly from a Gaussian distribution. In all four points of measurement the PDFs show extended "wings" representing increased probabilities of large amplitude fluctuations at scales smaller than $\tau_{G}=61.47 \mathrm{~s}$. The PDFs at scales larger than $\tau_{G}$ can be approximated by a Gaussian distribution, as illustrated by Fig. 5. This behavior of PDFs is interpreted as a hallmark of intermittency. If the Taylor hypothesis is satisfied, the threshold $\tau_{G}$ would correspond to a spatial scale of about $170 \mathrm{~km}$. We are aware that the assumption that the time fluctuations observed in satellite data correspond to spatial fluctuations may not be fully justified in the cusp. A preliminary analysis shows similarities between dynamic auto and cross correlation coefficients computed for pairs of the four Cluster satellites. This behavior has been previously considered as a verification that temporal fluctuations correspond to spatial variations (Weygand et al., 2005).

The flatness $\mathcal{F}$ also grows for $\tau_{G} \leq 60 \mathrm{~s}$, for all four Cluster spacecraft. The flatness parameter is, however, nearly equal to 3 for scales larger than $\tau_{G}$, confirming the absence of in-

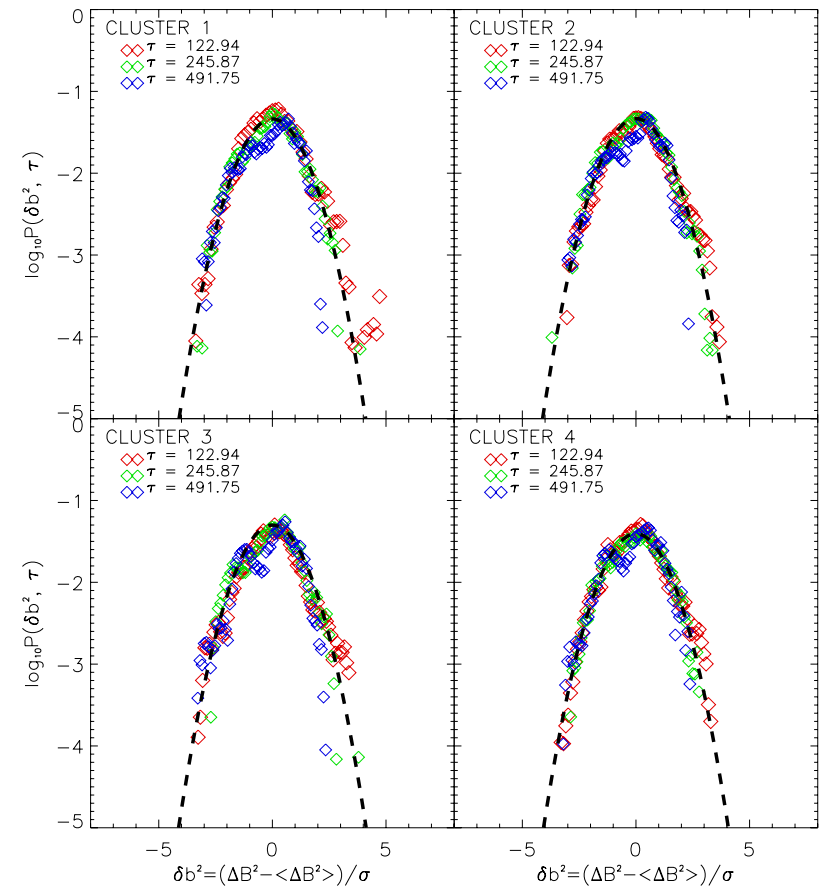

Fig. 5. PDFs of the magnetic energy fluctuations measured by the four Cluster satellites in the cusp at scales larger than $\tau_{G}=61,47 \mathrm{~s}$; see caption of Fig. 4.

termittency at a coarse-grained level. Quite surprisingly, the flatness decreases at the shortest scales, a behavior that we also observe in the PDFs (not shown for the smallest scales). The scale at which $\mathcal{F}$ starts to decrease vary with the satellite between $\tau \sim 0.3 \mathrm{~s}$ and $\tau \sim 1 \mathrm{~s}$. This might indicate that the PDFs tend again towards more Gaussian distributions. However we have to be careful with this interpretation because the flatness is related only to the fourth order moment of the fluctuations and higher order moments might behave differently. Only a multifractal analysis could help us answering this question. Furthermore on-board treatment of magnetometer data filters out part of the power at frequencies larger than $10 \mathrm{~Hz}$ (E. Lucek, private communication, 2007) which can also contribute to the reduction of $\mathcal{F}$ at smallest scales.

The PDFs describing the magnetic energy fluctuations observed by Cluster in the cusp do not rescale with (6). In the central part, corresponding to smaller amplitude fluctuations, the PDFs at all scales have the tendency to collapse on a single curve. Interestingly, this tendency is better seen in B-field data from Cluster 3 and 4, than in data from Cluster 1 and 2. For scales smaller than $\tau_{G}$ no collapse of the wings of PDFs toward a stable distribution (Gaussian or Levy) is observed for none of the four Cluster sattelites. The absence of selfsimilarity of PDFs of $B^{2}$ fluctuations is again an evidence of the intermittent process detected in the cusp.

The attempt to rescale the PDFs observed in the cusp with the mono-power law given in (2) is not successful. Indeed, 


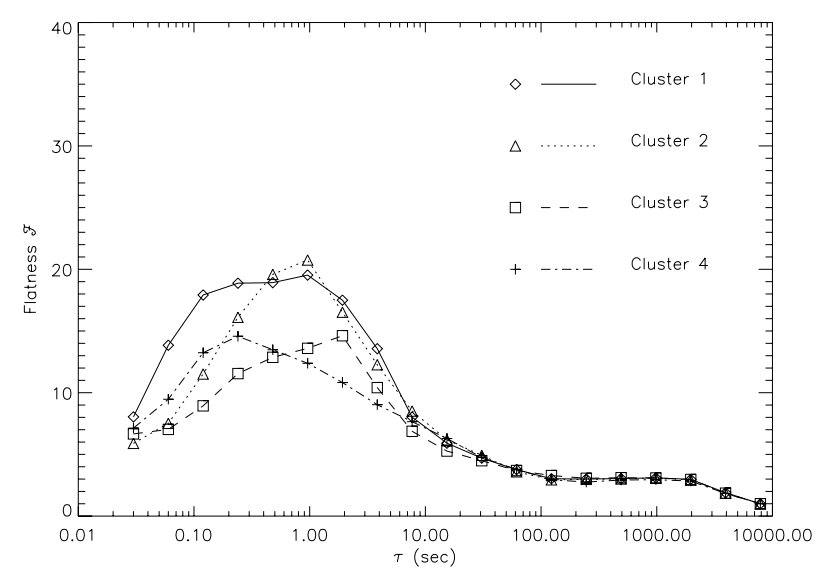

Fig. 6. Flatness $\mathcal{F}$ versus time scale $\tau$ for the magnetic energy fluctuations measured in the cusp by the four Cluster satellites.

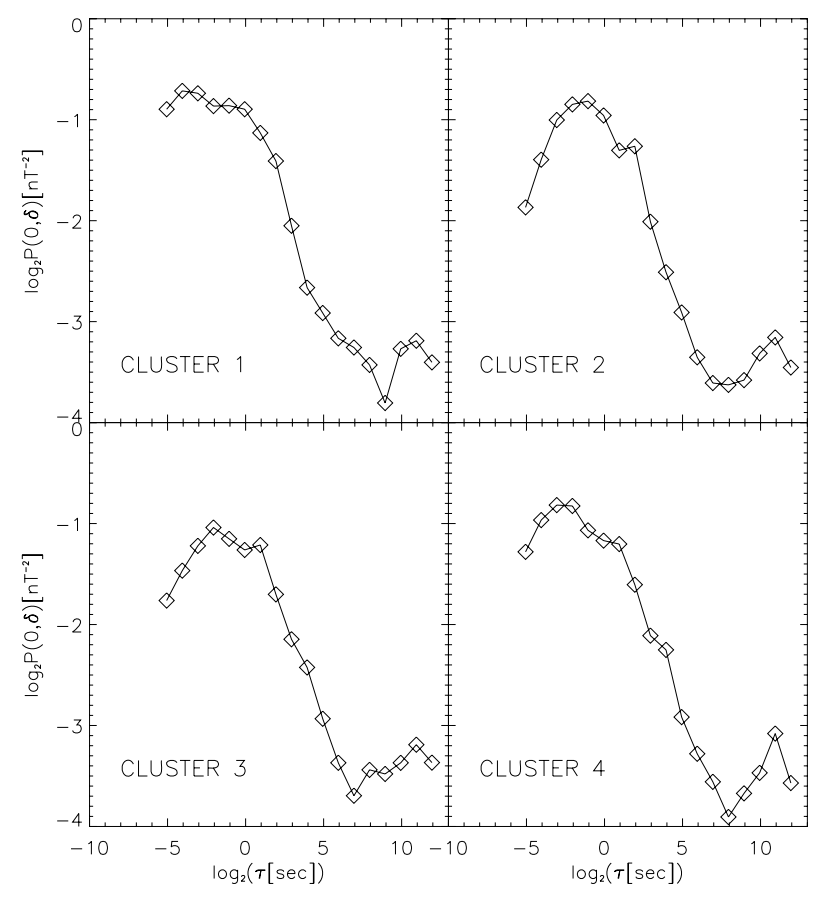

Fig. 7. Scaling of the peak of the unscaled PDFs of the magnetic energy fluctuations in the cusp region, for all Cluster spacecraft.

the scaling parameter $\alpha$ from (2) may be found from a linear fit of the variation of the maximum of unscaled PDFs, $P(0, \tau)$, with scale $\tau$ (Hnat et al., 2002). Alternatively, it could be derived by plotting the variance, $\sigma$, against scale $\tau$, or by a multifractal analysis. The variation of $P(0, \tau)$ with $\tau$ in the cusp is shown in Fig. 7. One can note a transition region from small $(\tau<1 \mathrm{~s})$ to large scales $\left(\tau>2^{7} \mathrm{~s}\right)$, that would correspond to the inertial range, where the variation of $P(0, \tau)$ with $\tau$ is not linear therefore the $\alpha$ parameter in (2) could not be determined appropriately and the one-parameter rescaling could not be achieved. A multifractal analysis of the fluctuations can give more insight on the structure of the energy cascade and will be the subject of a future paper.

The analysis of PDFs outlined above gives the image of a turbulent cusp with an anomalous scaling of the energy transfer. The PDFs indicate the presence of "events" that are at the origin of intermittency, i.e. large amplitude fluctuations over small spatial scales. A deeper insight on the turbulent process can be achieved if one could identify and localize the sudden large amplitude fluctuations and determine at which scales the energy transfer/dissipation is maximum. The wavelet analysis can unfold the structure of the signal both in space (or time $t$ ) and scale $\tau$. The local intermittency measure, $\operatorname{LIM}(\tau, t)$, defined in (5) characterizes the local activity of the analyzed field (magnetic energy in our case) at scale $\tau$ (Bruno et al., 2001). The Haar wavelet transform, based on Haar "mother" function (Haar, 1910),

$\Psi(x)=\left\{\begin{array}{rll}1 & , & 0<x<\frac{1}{2} \\ -1 & , & \frac{1}{2} \leq x<1 \\ 0 & , & \text { otherwise }\end{array}\right.$

is an appropriate tool to investigate the presence of discontinuities. Therefore it has been selected to compute the wavelet coefficients (4) for the fluctuations of $|B|^{2}$ measured by Cluster. The results obtained for Cluster 1 data are shown in Fig. 8. Data from all other three satellites, not shown here, were also analyzed and they lead to the same conclusions.

The wavelet analysis has been slightly extended to the left and to the right of interval (B). The two transitions, from the magnetosphere to the cusp and from the cusp to the magnetosheath, are clearly visible in the spectrum of wavelet coefficients, at 04:00 UT and 06:10 UT. The relative high values taken by the wavelet coefficients, $C(\tau, t)$, for large scales prior to 04:00 UT are due to the dipolar component of the magnetic field that decreases with altitude. In the magnetosheath ( $\mathrm{t}>06: 10 \mathrm{UT}$ ) we note the absence of wavelet power for scales smaller than approximately $32 \mathrm{~s}$. The spectrum of $C(\tau, t)$ in the cusp is characterized by "fingers" extending from large to small scales (panel $b$ of Fig. 8). Note that the "tilt" towards the right of the time axis of the "fingers" corresponding to active regions observed in Fig. 8 is not a physical effect but it is due to the intrinsic asymmetry of the Haar wavelet transform.

The values of $L I M$ (panel c of Fig. 8) identifies the "most intermittent" scales and regions. It is interesting to note that, although most of the wavelet power increases with scale, the $L I M$ coefficient takes values larger than 1.0, corresponding to intermittency (see definition 5), in a more limited range of scales, in general smaller than $65 \mathrm{~s}$. The latter value is close to the threshold $\tau_{G}$ obtained in the cusp for the transition of PDFs from non-Gaussian to Gaussian (see Fig. 4). At scales larger than $65 \mathrm{~s}$ the Haar wavelet power (panel b of Fig. 8) is partitioned roughly uniformly over the entire time interval, except for some regions evidenced by yellow "puffs" in 


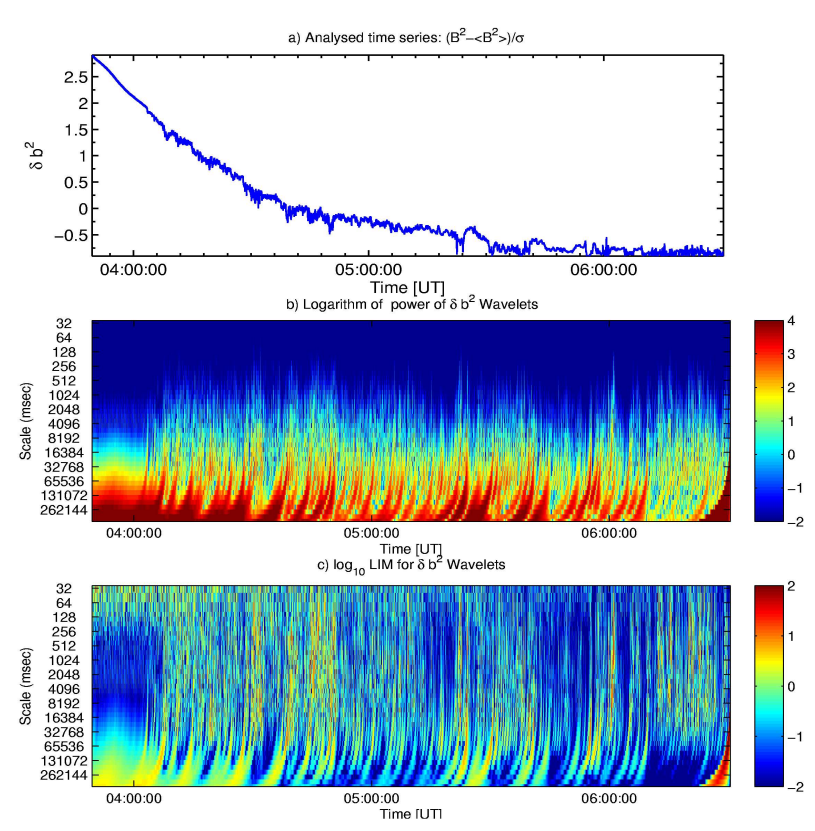

Fig. 8. Haar wavelet analysis of $\mathrm{B}^{2}$ fluctuations measured by Cluster-1: (a) the analyzed time series; (b) logarithm of the power of $\delta b^{2}$ wavelets computed with (4); (c) the local intermittency measure $(L I M)$ derived with (5).

panel c. One should note, however, that in those regions the LIM takes values of the order of 0.5 thus the fluctuations corresponding to scales larger than $65 \mathrm{~s}$ are not intermittent (Farge, 1992). At scales smaller than $65 \mathrm{~s}$ the intermittency is localized in several events, the most "intense" of them being observed at 04:30 UT, 04:36 UT, 04:42 UT, 04:48 UT, 05:21 UT, 05:24 UT, 05:29 UT, 05:56 UT, 06:00 UT, as illustrated in panel c of Fig. 8. During these events $L I M$ takes values larger than 1.0 over a range of scales from 0.1 to $65 \mathrm{~s}$. Thus the wavelet analysis reveals a picture of the cusp where intermixing of structures takes place over a broad range of scales. This aspect will be further discussed in the last section.

\subsection{Magnetosheath}

To conclude our study we give below a statistical analysis of the magnetic field fluctuations detected during the interval (C) of the orbit, when the Cluster satellites exit the cusp regions and surveyed the magnetosheath. The PDFs of the magnetic energy fluctuations rescaled with (6) are shown in Fig. 9. At small scales the PDFs are not Gaussian. At scales larger than $\tau_{G M}=15 \mathrm{~s}$ the PDFs tend to converge toward a Gaussian distribution, as seen in Fig. 10.

The intermittency in the magnetosheath is strong. The PDFs do not collapse on a master curve after rescaling. However in the central region, corresponding to small fluctuations, the PDFs do partially collapse onto the same curve,

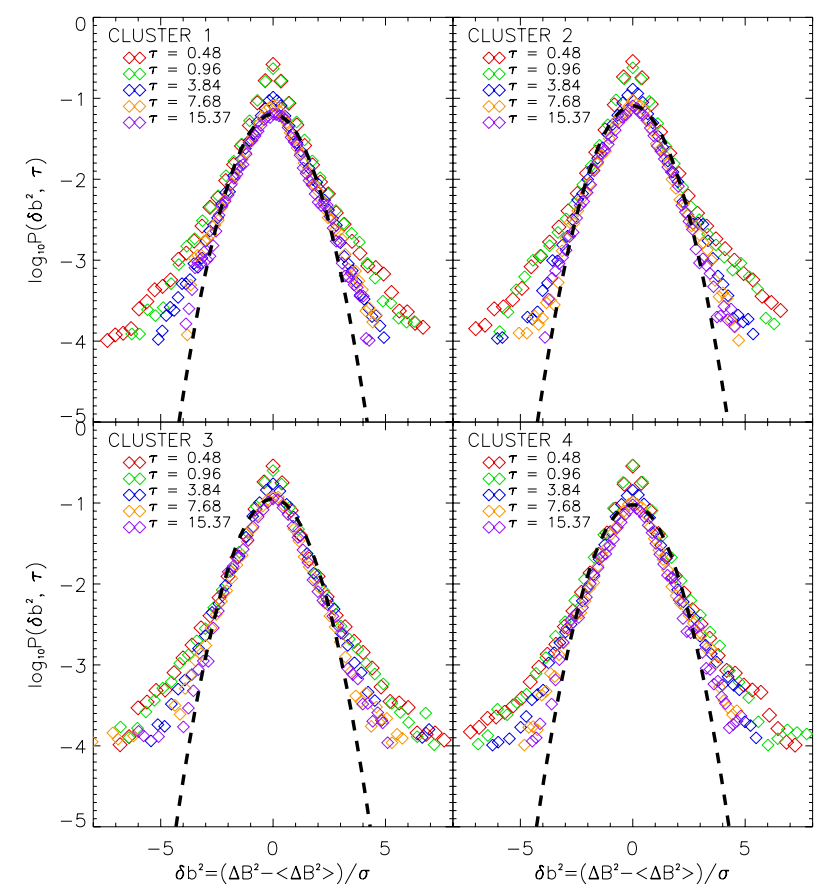

Fig. 9. PDFs of fluctuations of the magnetic energy measured by the four Cluster satellites in the magnetosheath. PDFs have been scaled with respect to their $\sigma(\tau)$.

a feature observed particularly in the data from Cluster 3 . The variation of $P(0, \tau)$ with $\tau$ (not shown) suggests that in the magnetosheath, as in the cusp, a mono-power scaling cannot be found, although at larger scales the PDFs seem to converge toward a stable distribution. This is confirmed by the flatness results given in Fig. 11: $\mathcal{F}$ strongly increases at smaller scales for the four Cluster spacecraft. On the other hand, at larger scales, the variation of $\mathcal{F}$ with the scale $\tau$ is flat and has values close to 3 , in agreement with the oneparameter scaling of the PDFs. The flatness starts to grow at a scale $\tau \sim 10 \mathrm{~s}$, again in agreement with the PDF analysis. We conjecture that the statistical properties of the magnetic energy fluctuations in the magnetosheath correspond to a turbulent process with characteristics different from the ones observed in the cusp. We note that the threshold scale separating non-Gaussian from Gaussian-like PDFs decreased from $65 \mathrm{~s}$ to about $15 \mathrm{~s}$, or, in spatial scales, from approximately 170 to about $35 \mathrm{~km}$.

The main driver of the turbulence both in the cusp and magnetosheath is the solar wind. Considering that the intermittency is related to energy transfer/dissipation processes taking place at the interface between coherent structures ( $\mathrm{Wu}$ and Chang, 2000) and assuming that these interfaces scale with the local proton Larmor radius, the decreasing of $\tau_{G}$ could be an effect of the decreasing of the proton temperature from the cusp to the magnetosheath. 


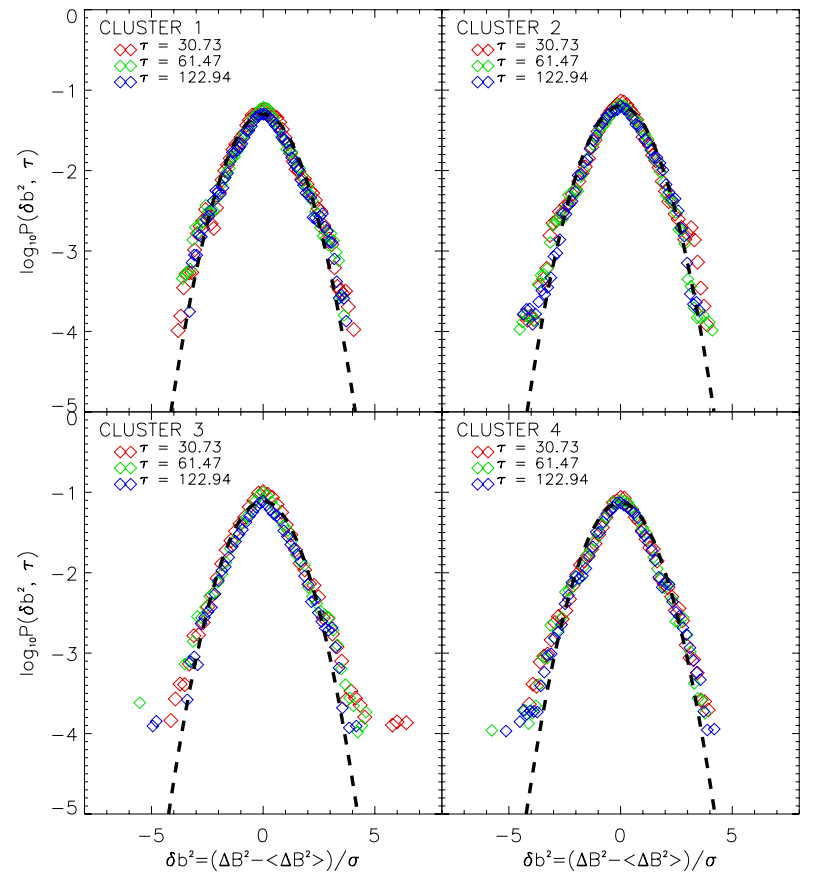

Fig. 10. PDFs of fluctuations of the magnetic energy measured by the four Cluster satellites in the magnetosheath for scales larger than $\tau_{G M}$.

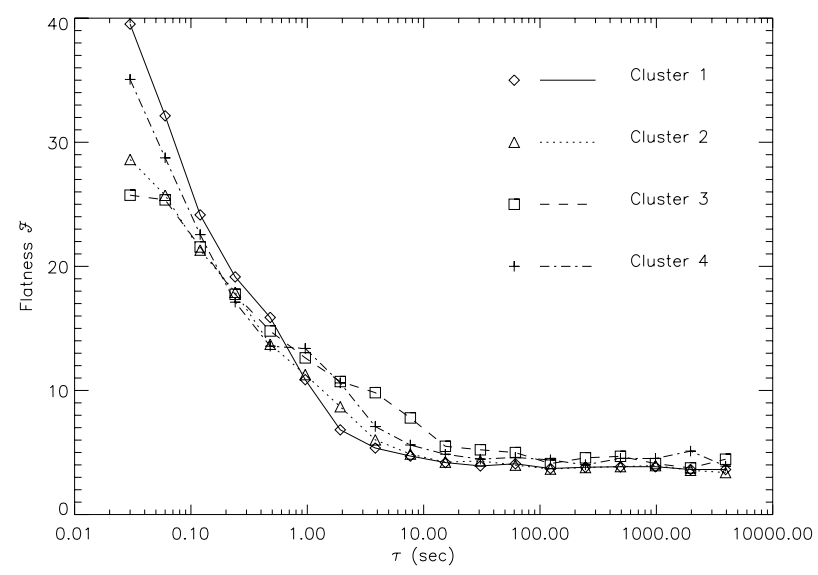

Fig. 11. Flatness $\mathcal{F}$ versus time scale $\tau$ for the magnetic energy fluctuations measured in the magnetosheath by the four Cluster satellites.

\section{Summary, conclusions}

There is increasing evidence that the terrestrial magnetosphere is a nonlinear system driven far from equilibrium by the continuous interaction with the solar wind that is often turbulent. One of the regions the most "exposed" to the turbulent solar wind are the magnetospheric cusps. The analysis of a Cluster orbit on 26 February 2001 allows us to study the statistical properties of magnetic field fluctuations in the cusp but also in the regions below (magnetospheric lobe) and above (magnetosheath). It is shown that in the magnetospheric lobe the fluctuations are random and are described by a Gaussian probability distribution function. In the cusp, however, the magnetic energy fluctuations show strong intermittency, with larger fluctuations having increased probabilities at scales smaller than $\tau_{G}=65 \mathrm{~s}$. The PDFs do not collapse when scaled with respect to their variance and the one-parameter rescaling procedure suggested by Hnat et al. (2002) could not be applied. The variation of the maximum of PDFs with scale suggests a multifractal process. We conjecture that the statistical properties of the fluctuations observed in the cusp are perhaps due to the turbulent transfer of the energy from driving to dissipation scales.

Magnetic field data from the "shocked" solar wind, in the terrestrial magnetosheath, were also analyzed. Our results indicate a change of the turbulent regime from the cusp to the magnetosheath. Although the PDFs in the magnetosheath keep their non-Gaussian characteristics for certain scales, they become Gaussian at scales smaller than in the cusp regions $\left(\tau_{G} \approx 15 \mathrm{~s}\right.$ or $\left.35 \mathrm{~km}\right)$. The decrease of $\tau_{G}$ from cusp to the magnetosheath may suggest that the intermittency is related to processes at the interface between coherent structures scaling with the local Larmor radius, like for instance tangential discontinuities (Bruno et al., 2001).

In this study, we give examples of magnetic fluctuations with properties that may perhaps be interpreted as the image of the spatial and temporal multiscale interactions between the solar wind and the magnetosphere. Investigations based on the probability distribution functions of the $B^{2}$ fluctuations of in-situ Cluster data in key magnetospheric regions such as the cusp indicate the presence of intermittency on a broad range of scales. An analysis of the flatness of the ensemble of magnetic energy fluctuations confirms the nonGaussian statistics at the same scales observed in PDFs. The LIM coefficient derived from a Haar wavelet transform of the signal identifies the "events" producing large amplitude fluctuations and the range of scales that concentrate most of the energy transfer. Such properties seem to suggest that the transfer of energy among various scales is nonuniform and non self-similar. It has been suggested (see e.g., reviews by Chang et al., 2004, 2006 and simulation results of $\mathrm{Wu}$ and Chang, 2000) that such intermittency of dynamical complexity is the manifestation of the stochastic interactions of coherent structures of various sizes that permeate throughout the anisotropic plasma medium. Recently, coherent structures of the form of Alfvén vortices have been detected using the Cluster observations both in the cusp (Sundkvist et al., 2005) and the magnetosheath (Alexandrova et al., 2006). In our future investigations, we shall plan to study the multifractal nature of the energy transfer in the cusp region and to investigate in more depth some of the queries partially addressed in this paper (such as inhomogeneity and non-stationarity). 
Acknowledgements. The authors would like to thank the Cluster Active Archive and E. Lucek, the PI of the FGM experiment on board CLUSTER, for providing high resolution magnetic field data. ME and HL acknowledge the support of the PRODEX-Cluster project conducted by BIRA-IASB Bruxelles and would like to thank S. Tam (CKU Taiwan) for his support with wavelet analysis. TC acknowledges partial support from AFOSR, NSF and NASA. ME aknowledges partial support from ESA's PECS project KEEV.

Edited by: P.-L. Sulem

Reviewed by: R. Bruno and M. Leubner

\section{References}

Alexandrova, O., Mangeney, A., Maksimovici, M., et al.: Alfvén vortex filaments observed in the magnetosheath downstream of a quasi-perpendicular bow shock, J. Geophys. Res., 111, A12208, doi:10.1029/2006JA011934, 2006.

Balogh, A., Carr, C. M., Acuna, M. H., et al.: The Cluster Magnetic Field Instrument: overview of in-flight performance and initial results, Ann. Geophys., 19, 1207-1217, 2001, http://www.ann-geophys.net/19/1207/2001/.

Bruno, R., Carbone, V., Veltri, P., et al.: Identifying intermittency events in the solar wind, Planet. Space Sci., 49(12), 1201-1210, 2001.

Bruno, R., Carbone, V., Sorriso-Valvo, L., and Bavassano, B.: Radial evolution of solar wind intermittency in the inner heliosphere, J. Geophys. Res., 108(A3), A1130, doi:10.1029/2002JA009615, 2003.

Burlaga, L. F.: Intermittent turbulence in the solar wind, J. Geophys. Res., 96(A4), 5847-5851, 1991.

Burlaga, L. F. and F.-Vinãs, A.: Multiscale structure of the magnetic field and speed at $1 \mathrm{AU}$ during the declining phase of solar cycle 23 described by a generalized Tsallis probability distribution function, J. Geophys. Res., 109, A12107, doi:10.1029/2004JA010763, 2004.

Castaing, B., Gagne, Y., and Hopfinger, E. J.: Velocity probability density functions of high Reynolds number turbulence, Physica D, 46, 177-200, 1990.

Consolini G., Chang T., and Lui A. T. Y.: Complexity and topological disorder in the Earth's magnetotail dynamics, in nonequilibrium phenomena in plasmas, edited by: Sharma, A. S. and Kaw, P. K., Springer, the Netherlands, 51, 2005.

Chang, T.: Multiscale intermittent turbulence in the magnetotail, Substorms-4 : International Conference on Substorms-4, Lake Hamana, Japan: March 9-13, 1998, edited by: Kokubun, S., and Kamide, Y., Dordrecht, London, Kluwer Academic Publishers, 431, 1998

Chang, T., Tam, S. W. Y., and Wu, C.-C.: Complexity induced anisotropic bimodal intermittent turbulence in space plasmas, Phys. Plasmas, 11(4), 1287, 2004.

Chang, T., Tam, S. W. Y., and Wu, C.-C.: Complexity in Space Plasmas - A Brief Review, Space Sci. Rev., 122(1-4), 281-291, 2006.

Daubechies, I.: Ten lectures on wavelets, Society for industrial and applied mathematics, Philadelphia, 53-105, 1992.

Farge, M., Holschneider, M., and Colonna, J. F.: Wavelet analysis of coherent structures in two-dimensional turbulent flows, in Topo- logical fluid mechanics, edited by: Moffat, H. K., Cambridge University Press, Cambridge, 765-776, 1990.

Farge, M.: Wavelet transforms and their application to turbulence, Annu. Rev. Fluid Mech., 24, 395-457, 1992.

Frisch, U.: Turbulence, Cambridge University Press, Cambridge, 120-123, 1995.

Haar, A.: Zur theorie der orthogonalen Functionen-Systeme, Math. Ann., 69, 331-371, 1910.

Hnat, B., Chapman, S. C., Rowlands, G., et al.: Finite size scaling in the solar wind magnetic field energy density as seen by WIND, Geophys. Res. Lett., 29(10), 14446, doi:10.1029/2001GL014587, 2002.

Hnat, B., Chapman, S. C., and Rowlands, G.: Scaling and a FokkerPlanck model for fluctuations in geomagnetic indices and comparison with solar wind $\epsilon$ as seen by Wind and ACE, J. Geophys. Res., 110(A8), A08206, doi:10.1029/2004JA010824, 2005.

Leubner M. P.: Fundamental issues on kappa-distributions in space plasmas and interplanetary proton distributions, Phys. Plasmas, 11, 1308-1316, 2004.

Leubner, M. P. and Vörös, Z.: A nonextensive entropy approach to solar wind intermittency, Ap. J., 618, 547-555, 2005.

Lui, A. T. Y.: Multifractal and intermittent nature of substormassociated magnetic turbulence in the magnetotail, J. Atmos. SolTerr. Phy., 63(13), 1379-1385, 2001.

Lundin, R., Sauvaud, J.-A., Rme, H. et al., Evidence for impulsive solar wind plasma penetration through the dayside magnetopause, Ann. Geophys., 21(2), 457-472, 2003.

Matthaeus, W. H. and Goldstein, M. L.: Measurement of the rugged invariants of magnetohydrodynamic turbulence in the solar wind, J. Geophys. Res., 87(A8), 6011-6028, 1982.

March, E and C.Y. Tu, Non-Gaussian probability distributions of solar wind fluctuations, Ann. Geophys., 12, 1127-1138, 1994, http://www.ann-geophys.net/12/1127/1994/.

Meneveau, C., Analysis of turbulence in the orthonormal wavelet representation, J. Fluid Mech., 232, 469-520, 1991.

Nykyri, K., Cargill, P. J., Lucek, E., et al.: Cluster observations of magnetic field fluctuations in the high-altitude cusp, Ann. Geophys., 22, 2413-2429, 2004, http://www.ann-geophys.net/22/2413/2004/.

Nykyri, K., Grison, B., Cargill, P. J., et al.: Origin of the turbulent spectra in the high-altitude cusp: Cluster spacecraft observations, Ann. Geophys., 24(3), 1057-1075, 2006.

Pagel, C. and Balogh, A.: A study of magnetic fluctuations and their anomalous scaling in the solar wind: the Ulysses fast-latitude scan, Nonlin. Processes Geophys., 8(4/5), 313-330, 2001.

Pickett, J. S., Menietti, J. D., Dowell, J. H. et al., Polar spacecraft observations of the turbulent outer cusp/magnetopause boundary layer of Earth, Nonlin. Processes Geophys., 6(3/4), 195-204, 1999.

Pickett, J. S., Menietti, J. D., Hospodarsky, G. B., Gurnett, D. A., and Stasiewicz, K.: Analysis of the turbulence observed in the outer cusp turbulent boundary layer, Adv. Space Res., 30(12), 2809-2814, 2002.

Ruzmaikin, A. A., Feynman, J., Goldstein, B. E., and Smith, E. J.: Intermittent turbulence in solar wind from the south polar hole, J. Geophys. Res., 100(A3), 3395-3403, 1995.

Savin, S. P., Borodkova, N. L., Budnik, E. Y., Federov, A. O., Klimov, S. I., et al.: Interball Tail Probe measurements in outer cusp and boundary layers, in Geospace mass and energy flow: 
results from the International Solar-Terrestrial Physics Program, edited by: Horwitz, J. L., Gallagher, D. L., and Peterson, K. W., Geophysical Monograph 104, 25-44, American Geophysical Union, Washington D.C., 1998.

Savin, S., Büchner, J., Consolini, G., et al.: On the properties of turbulent boundary layer over polar cusps, Nonlin. Processes Geophys., 9(5/6), 443-451, 2002.

Sorriso-Valvo,L., Carbone,V., Giuliani,P., Veltri,P., Bruno,R., Antoni,V., and Martines,E.: Intermittency in plasma turbulence, Planet. Space Sci., 49, 12, 1193-1200, 2001.

Sornette, D.: Critical phenomena in natural sciences, SpringerVerlag Berlin Heidelberg, 79-95, 2000.

Sundkvist, D., Krasnoselskikh, V., Shukla, P. K., et al.: In situ multi-satellite detection of coherent vortices as a manifestation of Alfvén turbulence, Nature, 436, 825-828, 2005.

Tam, S. W. Y., Chang, T., Kintner, P. M., and Klatt, E.: Intermittency analyses on the SIERRA measurements of the electric field fluctuations in the auroral zone, Geophys. Res. Lett., 32, L05109, doi:10.1029/2004GL021445, 2005.

Taylor, G. I.: The spectrum of turbulence, Proc. R. Soc. Lon. Ser-A, 164, 476, 1938.

Taylor, M. G. G. T., Dunlop, M. W., Lavraud, B., et al.: Cluster observations of a complex high-altitude cusp passage during highly variable IMF, Ann. Geophys., 22(10), 3707-3719, 2004.
Torrence, C. and Compo, G. P.: A practical guide to wavelet analysis, B. Am. Meteor. Soc., 79(1), 61-78, 1998.

Tsyganenko, N. A.: Modeling the Earth's magnetospheric magnetic field confined within a realistic magnetopause, J. Geophys. Res. 100(A4), 5599-5612, 1995.

Tsyganenko, N. A. and Russell, C. T.: Magnetic signatures of the distant polar cusps: Observations by Polar and quantitative modeling, J. Geophys. Res., 104(A11), 24 939-24 955, 1999.

Vörös, Z., Baumjohann, W., Nakamura, R., et al.: Multi-scale magnetic field intermittence in the plasma sheet, Ann. Geophys., 21 , 1955-1964, 2003, http://www.ann-geophys.net/21/1955/2003/.

Weygand, J. M., Kivelson, M. G., Khurana, K. K., et al.: Plasma sheet turbulence observed by Cluster II, J. Geophys. Res., 110, A01205, doi:10.1029/2004JA010581, 2005.

Wu, C. C. and Chang, T.: 2D MHD Simulation of the Emergence and Merging of Coherent Structures, Geophys. Res. Lett., 27(6), 863-866, 2000.

Yordanova, E., Grzesiak, M., Wernik, A. W., et al.: Multifractal structure of turbulence in the magnetospheric cusp, Ann. Geophys., 22, 2431-2440, 2004, http://www.ann-geophys.net/22/2431/2004/.

Yordanova, E., Bergman, J., Consolini, G., et al.: Anisotropic scaling features and complexity in magnetospheric-cusp: a case study, Nonlin. Processes Geophys., 12, 817-825, 2005, http://www.nonlin-processes-geophys.net/12/817/2005/. 